EGU2020-8106

https://doi.org/10.5194/egusphere-egu2020-8106

EGU General Assembly 2020

(c) Author(s) 2020. This work is distributed under

the Creative Commons Attribution 4.0 License.

\title{
Disentangling the effects of particles and circulation on 231 Pa/230Th during Heinrich Stadials
}

\author{
Jörg Lippold ${ }^{1}$, Finn Süfke ${ }^{1}$, Jens Grützner ${ }^{2}$, and Frerk Pöppelmeier ${ }^{3}$ \\ ${ }^{1}$ Institute of Earth Sciences, Heidelberg University, Heidelberg, Germany (joerg.lippold@geow.uni-heidelberg.de) \\ ${ }^{2}$ Geosciences/Geophysics, Alfred Wegener Institute, Bremerhaven, Germany (Jens.Gruetzner@awi.de) \\ ${ }^{3}$ Climate and Environmental Physics, University of Bern, Bern, Switzerland (frerk.poeppelmeier@climate.unibe.ch)
}

It has been shown that during Heinrich stadials northern deep water production ceased leading to an enhanced inflow of southern sourced water. Although Heinrich events are not considered to represent the primary trigger of Heinrich stadials the reorganisation of Atlantic ocean dynamics during their occurrences is an active field of research. In particular, Heinrich stadial 2 (HS2) is of high interest, based on the observation that the interplay with the climate system was very different during HS2 compared to HS1, although the magnitude of iceberg and freshwater discharge was similar (Hemming, 2004). During HS2 sea-level was still decreasing while the atmospheric $\mathrm{CO}_{2}$ content was relatively stable unlike the climatic evolution during Heinrich HS1.

The notion of a reduced Atlantic Meridional Overturning Circulation (AMOC) during Heinrich Stadials is mainly strengthened by the ${ }^{231} \mathrm{~Pa} /{ }^{230} \mathrm{Th}$ records from the Bermuda Rise. However, other influencing factors, capable of increasing the sedimentary ${ }^{231} \mathrm{~Pa} /{ }^{230} \mathrm{Th}$ without according decreases in AMOC strength, need to be considered as well. Besides biogenic opal, high dust fluxes may also result in enhanced scavenging rate of both radionuclides and consequently higher sedimentary ${ }^{231} \mathrm{~Pa} /{ }^{230} \mathrm{Th}$ signals, since another distinct feature that accompanies Heinrich Stadials is the high atmospheric concentration of dust in the northern hemisphere. Furthermore, high dust concentrations might be an indicator of a vigorous wind system and therefore strong ocean mixing, which can lead to the enhanced formation of nepheloid layers These layers are suspected to cause strong bottom scavenging and consequently high sedimentary ${ }^{231} \mathrm{~Pa} /{ }^{230} \mathrm{Th}$. Very high dust fluxes were observed e.g. during HS2 and MIS4. Here, we compare ${ }^{231} \mathrm{~Pa} /{ }^{230} \mathrm{Th}$ with dust records in order to disentangle the effects of scavenging and circulation on the recorded sedimentary ${ }^{231}$ $\mathrm{Pa} /{ }^{230} \mathrm{Th}$ from the northwestern Atlantic. 